\title{
MÉTOdo DE DIFERENCIAS FINITAS EN EL DOMINIO DE LAS FRECUENCIAS PARA CRISTALES FOTÓNICOS 1D Y 2D
}

\author{
JUAN P. VASCO ${ }^{1}$ \\ Herbert Vinck-Posada ${ }^{2}$
}

\section{Resumen}

En este trabajo se estudian los modos electromagnéticos en cristales fotónicos uno-dimensionales y dos-dimensionales (1D y 2D) a través del método de diferencias finitas en el dominio de las frecuencias FDFD. Los diagramas de bandas son calculados para cristales 1D regulares y con defecto, al igual que sus perfiles de intensidad electromagnética. De igual manera se calculan los diagramas de bandas y perfiles de intensidad para cristales $2 \mathrm{D}$ regulares en redes hexagonal y cuadrada. Nuestros cálculos son comparados con los obtenidos en el software MPB proporcionado por el MIT y basado en el método de expansión de ondas planas.

\section{Palabras clave}

Cristal fotónico, defecto 1D, red hexagonal, red cuadrada.

\section{Abstract}

In this work we study the electromagnetic modes in onedimensional and two-dimensional photonic crystals through finite difference frequency domain method. The band diagrams and the intensity profiles are calculated for one-dimensional photonic crystal with a defect, and for two-dimensional photonic crystals

1 Universidad de Antioquia, Grupo de Física Atómica y Molecular, jpvasco@gmail. com

2 Universidad Nacional de Colombia sede Bogotá, hvinckp@unal.edu.co

Fecha de recepción: 15 de marzo de 2010 Fecha de aceptación: 26 de junio de 2010 
[126] Método de diferencias finitas en el dominio de las frecuencias para cristales fotónicos...

in hexagonal and square lattices. Our calculations are compared with simulations made with MPB software based on plane wave expansion method.

\section{Keywords}

Photonic crystal, 1D defect, hexagonal lattice, square lattice. 


\section{INTRODUCCIÓN}

Durante las dos últimas décadas el estudio de nanodispositivos y metamateriales ha cobrado gran interés debido a sus prometedoras aplicaciones, que van desde el estudio de física fundamental, pasando por la ciencia de los materiales, hasta aplicaciones en el campo de las telecomunicaciones e industria (Chen et al., 2010). Los cristales fotónicos, que son sistemas cuya función dieléctrica es periódica en el espacio, son un importante ejemplo de este tipo de dispositivos, que si bien tienen un principio de funcionalidad simple (la periodicidad), producen importantes y atractivos efectos en el flujo de la radiación electromagnética (Joannopoulos et al., 1997). Uno de los más importantes es la aparición de band-gaps o regiones "prohibidas" en su relación de dispersión (conocida como diagrama de bandas), por lo tanto, una onda electromagnética con energía en dicha brecha energética será totalmente reflejada y sufrirá un rápido amortiguamiento en el cristal.

A tal fenómeno de reflexión, que no es de la misma naturaleza al producido en una superficie metálica, se le conoce como reflexión de Bragg distribuida. La introducción de defectos en cristales fotónicos, sean puntuales, lineales o planares, producen la localización de modos electromagnéticos o guiado de la radiación (Joannopoulos et al., 2008), y es en este ámbito donde los cristales fotónicos adquieren gran relevancia, pues con dichos sistemas es posible producir microcavidades con elevados factores de calidad y guías de onda de alta eficiencia (Vahala, 2003). Los cristales fotónicos pueden ser descritos formalmente con la teoría electromagnética de Maxwell, lo que los convierte en sistemas de características escalables, es decir, las propiedades electromagnéticas de un cristal microscópico cuyo trabajo sea en el rango espectral visible, se mantienen si el cristal se escala a un tamaño macroscópico en el rango de las microondas. Lo que los convierte en sistemas de fácil acceso experimental.

Es posible resolver las ecuaciones de Maxwell a través de una gran variedad de métodos, tanto analíticos como numéricos, para un cristal fotónico, entre ellos encontramos expansión en ondas 
[128] Método de diferencias finitas en el dominio de las frecuencias para cristales fotónicos...

planas PWE (Sakoda, 2005), matriz de transferencia TM (Yariv $\&$ Yeh, 2007), diferencias finitas en el dominio de las frecuencias FDFD y del tiempo FDTD (Taflove \& Hagness, 2000), entre otros. En este trabajo se estudia el método FDFD en cristales de periodicidad unidimensional y bidimensional, de la misma manera se exponen sus fortalezas a la hora de implementarlo en un sistema electromagnético como lo es un cristal fotónico.

\section{MÉTOdO TEÓRICO}

El método de diferencias finitas consiste en aproximar las derivadas de una ecuación diferencial a diferencias finitas, este hecho determina un grid o malla espacial (en el caso en que las variables sean de posición) que cuanto más fino sea mejor será la aproximación, en principio, a la ecuación diferencial en sí. En el caso particular de sistemas electromagnéticos, la evaluación de campos eléctricos y magnéticos en el mismo punto puede llevar a que las ecuaciones de divergencia de Maxwell no se cumplan en ausencia de cargas y corrientes, por lo que se debe recurrir a un tipo especial de grid llamado grid de Yee (Yee, 1966).

Este tipo de malla, mostrada en la Fig. 1, se construye de forma tal que los campos eléctrico y magnético sean evaluados en puntos diferentes, dicha elección asegura que los campos sean libres de divergencia, que la aproximación a diferencia finita tenga una precisión mayor que la usual y que puedan estudiarse los campos eléctrico y magnético en una misma simulación (Taflove \& Hagness, 2000). Cuando el sistema es libre de cargas y corrientes las ecuaciones de divergencia son una consecuencia directa de las ecuaciones rotacionales de Maxwell, por lo tanto sólo se realizará la discretización de estas últimas.
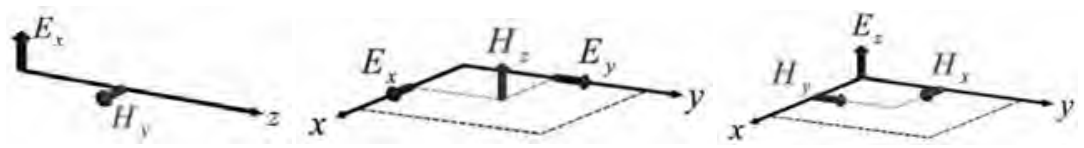

Fig. 1. Campos eléctrico y magnético evaluados en Un GRID DE Yee de UN Sistema 1D Y 2D RESPECTIVAMENTE 
Bajo las aproximaciones de medios lineales y no magnéticos, ausencia de fuentes de carga y de corriente, además de una dependencia armónica en el tiempo (fase estacionaria), las ecuaciones rotacionales de Maxwell se reducen a:

$$
\begin{aligned}
& \nabla \times \vec{H}(\vec{r})=-i \frac{\omega}{c} \epsilon(\vec{r}) \vec{E}(\vec{r}) \\
& \nabla \times \vec{E}(\vec{r})=i \frac{\omega}{c} \vec{H}(\vec{r})
\end{aligned}
$$

Donde se ha asumido una dependencia espacial de la función dieléctrica y una dependencia temporal de la forma $e^{-i \omega t}$. Note que en el caso $2 \mathrm{D}$ el confinamiento del vector de onda en el plano $x y$ divide las polarizaciones posibles de los modos del sistema en dos: caso transversomagnético (TM) y caso transversoeléctrico (TE). En el primer caso el campo eléctrico es perpendicular al plano de propagación, mientras que en el segundo caso es el vector de campo magnético el que hereda esta perpendicularidad. Las ecuaciones (1) y (2) pueden ser escritas para el sistema 1D asumiendo propagación en dirección $x$, y campos eléctrico y magnético sobre los ejes z y y respectivamente:

$$
\begin{aligned}
& \frac{\partial H_{y}}{\partial x}=-i \frac{\omega}{c} \epsilon(x) E_{z} \\
& \frac{\partial E_{z}}{\partial x}=-i \frac{\omega}{c} H_{y}
\end{aligned}
$$

De la misma forma para el sistema bidimensional (1) y (2) se reducen en el caso TM a las siguientes expresiones:

$$
\begin{aligned}
& \frac{\partial E_{z}}{\partial y}=i \frac{\omega}{c} H_{x} \\
& \frac{\partial E_{z}}{\partial x}=-i \frac{\omega}{c} H_{y}
\end{aligned}
$$


$\frac{\partial H_{y}}{\partial x}-\frac{\partial H_{x}}{\partial y}=-i \frac{\omega}{c} \epsilon(x, y) E_{z}$

Y para el caso TE las expresiones toman la forma:

$$
\begin{aligned}
& \frac{\partial H_{z}}{\partial y}=-i \frac{\omega}{c} \epsilon(x, y) E_{x} \\
& \frac{\partial H_{z}}{\partial x}=i \frac{\omega}{c} \epsilon(x, y) E_{y} \\
& \frac{\partial E_{y}}{\partial x}-\frac{\partial E_{x}}{\partial y}=i \frac{\omega}{c} H_{z}
\end{aligned}
$$

Este conjunto de ecuaciones diferenciales puede ser aproximado a un conjunto de ecuaciones en diferencias finitas para un grid de Yee (Taflove \& Hagness, 2000), las expresiones para el sistema 1D (3) y (4) toman la forma:

$$
\begin{aligned}
& \boldsymbol{D}_{x}^{H_{y}} \boldsymbol{H}_{y}=\frac{H_{y}^{j}-H_{y}^{j-1}}{\Delta x}=-i \frac{\omega}{c} \epsilon^{m} E_{z}^{m}=-i \frac{\omega}{c} \boldsymbol{\epsilon} \boldsymbol{E}_{z} \\
& \boldsymbol{D}_{x}^{E_{z}} \boldsymbol{E}_{z}=\frac{E_{z}^{m+1}-E_{z}^{m}}{\Delta x}=-i \frac{\omega}{c} H_{y}^{j}=-i \frac{\omega}{c} \boldsymbol{H}_{y}
\end{aligned}
$$

Donde los superíndices $m, m+1, j, j-1$, denotan los respectivos campos evaluados en los puntos $m \triangle x,(m+1) \triangle x, j \triangle x,(j-1) \triangle x, y$ las matrices $\boldsymbol{D}_{x}^{H_{y}}$ y $\boldsymbol{D}_{x}^{E_{z}}$ aplicadas sobre los vectores $\boldsymbol{H}_{y}$ y $\boldsymbol{E}_{z}$, reproducen el sistema de ecuaciones en diferencias finitas (Rumpf, 2006). En el caso bidimensional TM, la aproximación a diferencias finitas de (5)-(7) toma la forma:

$$
\begin{aligned}
& \boldsymbol{D}_{y}^{E_{z}} \boldsymbol{E}_{z}=\frac{E_{z}^{m, j+1}-E_{z}^{m, j}}{\Delta y}=i \frac{\omega}{c} H_{x}^{m, j}=i \frac{\omega}{c} \boldsymbol{H}_{x} \\
& \boldsymbol{D}_{x}^{E_{z}} \boldsymbol{E}_{z}=\frac{E_{z}^{m+1, j}-E_{z}^{m, j}}{\Delta x}=-i \frac{\omega}{c} H_{y}^{m, j}=-i \frac{\omega}{c} \boldsymbol{H}_{y}
\end{aligned}
$$




$$
\begin{gathered}
\boldsymbol{D}_{x}^{H_{y}} \boldsymbol{H}_{y}-\boldsymbol{D}_{y}^{H_{x}} \boldsymbol{H}_{x}=\frac{H_{y}^{m, j}-H_{y}^{m-1, j}}{\Delta x}-\frac{H_{x}^{m, j}-H_{x}^{m, j-1}}{\Delta y} \\
=-i \frac{\omega}{c} \epsilon^{m, j} E_{z}^{m, j}=-i \frac{\omega}{c} \boldsymbol{\epsilon} \boldsymbol{E}_{z}
\end{gathered}
$$

Donde los superíndices, al igual que en el caso 1D, denotan las respectivas evaluaciones de los campos en el grid espacial, y las matrices $\boldsymbol{D}$ aplicadas sobre los campos reproducen el sistema de ecuaciones en diferencias finitas. El caso TE se discretiza de la misma forma que el TM. Las expresiones matriciales para el caso 1D y $2 \mathrm{D}$ pueden manipularse para desacoplar los campos y obtener una ecuación de onda matricial en términos de un sólo campo, el procedimiento es similar al seguido para desacoplar los campos en las ecuaciones diferenciales de Maxwell y obtener la ecuación de onda. Estas expresiones matriciales son:

$$
\begin{aligned}
& \boldsymbol{\epsilon}^{-\mathbf{1}} \boldsymbol{D}_{x}^{H_{y}} \boldsymbol{D}_{x}^{E_{z}} \boldsymbol{E}_{z}=-\left(\frac{\omega}{c}\right)^{2} \boldsymbol{E}_{z} \text { Caso 1D } \\
& \boldsymbol{\epsilon}^{-\mathbf{1}}\left(\boldsymbol{D}_{x}^{H_{y}} \boldsymbol{D}_{x}^{E_{z}}+\boldsymbol{D}_{y}^{H_{x}} \boldsymbol{D}_{y}^{E_{z}}\right) \boldsymbol{E}_{z}=-\left(\frac{\omega}{c}\right)^{2} \boldsymbol{E}_{z} \text { Caso 2D TM }
\end{aligned}
$$

Las ecuaciones (16) y (17) determinan las resonancias electromagnéticas en un sistema $1 \mathrm{D}$ y $2 \mathrm{D}$, sólo cuando la matriz $\boldsymbol{\epsilon}$ representa la discretización de la función dieléctrica de un material periódico dichas ecuaciones determinan estas resonancias en un cristal fotónico. Estas ecuaciones definen un problema de autovalores ordinario, que puede ser solucionado diagonalizando las matrices correspondientes. Es de gran importancia imponer de forma adecuada las condiciones de frontera a (16) y (17), que para un cristal fotónico son las condiciones de frontera de Bloch.

En analogía al transporte electrónico en un potencial periódico, tal y como se estudia en un cristal electrónico, los cristales fotónicos también pueden ser descritos a través de una teoría de bandas, espacios reales, recíprocos y zonas de Brillouin (Lourtioz, 2005). Por lo tanto, un estado electromagnético en un cristal fotónico puede ser escrito como un estado de Bloch (Kittel, 1996), que el es 
producto de una función periódica y una onda plana que juega el papel de envolvente:

$$
\boldsymbol{H}_{n k}(\vec{r})=e^{i \vec{k} \cdot \vec{r}} \boldsymbol{H}_{n k}^{p}(\vec{r})
$$

Donde $\boldsymbol{H}_{n k}^{p}(\vec{r})$ es una función periódica que depende de los índices de banda $n$ y vector de onda $k$. En virtud de la periodicidad del cristal, el estudio de sus propiedades físicas se reduce al estudio de la mínima porción que contiene toda la información de la estructura y en algunos casos contiene las simetrías de ésta. Cuando esta porción no contiene dichas simetrías es llamada celda unitaria y cuando las contiene es llamada celda unitaria de Wigner-Seitz. La celda unitaria en el espacio recíproco o espacio de es llamada primera zona de Brillouin, y es en esta zona donde se halla la relación de dispersión o diagrama de bandas del cristal fotónico. La condición de frontera de Bloch viene determinada por la relación (10). Si $\boldsymbol{H}_{n k}^{p}(\vec{r})$ es una función periódica de periodo $\vec{R}$ entonces:

$$
\boldsymbol{H}_{n k}(\vec{r}+\vec{R})=e^{i \vec{k} \cdot(\vec{r}+\vec{R})} \boldsymbol{H}_{n k}^{p}(\vec{r}+\vec{R})=e^{i \vec{k} \cdot \vec{R}} \boldsymbol{H}_{n k}(\vec{r})
$$

\section{SOLUCión DEL MODELO}

La matriz asociada al problema de autovalores de (16) $\boldsymbol{\epsilon}^{-\mathbf{1}} \boldsymbol{D}_{x}^{H_{y}} \boldsymbol{D}_{x}^{E_{z}}$ se construye en la celda unitaria del cristal 1D cuya longitud es $a_{1}$, ésta se muestra en la Fig. 2.

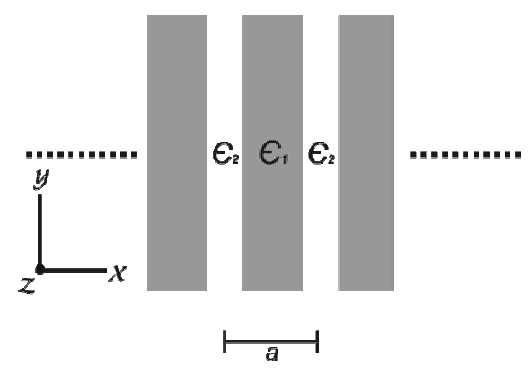

Fig. 2. CRISTAL FOTÓNICO 1D CON CELDA UNITARIA DE LONGITUD $a$ 
Las matrices $\boldsymbol{D}_{x}^{H_{y}}, \boldsymbol{D}_{x}^{E_{z}}, H_{y}$ y $\boldsymbol{\epsilon}$ asociadas a un grid de cuatro puntos son las siguientes:

$$
\begin{gathered}
\boldsymbol{D}_{x}^{H_{y}}=\frac{1}{\Delta x}\left(\begin{array}{cccc}
1 & 0 & 0 & -e^{-i k_{x} a} \\
-1 & 1 & 0 & 0 \\
0 & -1 & 1 & 0 \\
0 & 0 & -1 & 1
\end{array}\right) \boldsymbol{H}_{y}=\left(\begin{array}{l}
H_{y}^{1} \\
H_{y}^{2} \\
H_{y}^{3} \\
H_{y}^{4}
\end{array}\right) \\
\boldsymbol{D}_{x}^{E_{z}}=-\left(\boldsymbol{D}_{x}^{H_{y}}\right)^{\dagger} \\
\boldsymbol{\epsilon}=\left(\begin{array}{cccc}
\epsilon^{1} & 0 & 0 & 0 \\
0 & \epsilon^{2} & 0 & 0 \\
0 & 0 & \epsilon^{3} & 0 \\
0 & 0 & 0 & \epsilon^{4}
\end{array}\right)
\end{gathered}
$$

Donde $\epsilon^{i}=\epsilon(i \Delta x), H_{y}^{i}=H_{y}(i \Delta x)$ y $e^{-i k_{x} a}$ representa la condición de frontera de Bloch (19). Cuando se estudian cristales 2D los tipos de estructuras periódicas son variadas, en este trabajo se estudian en particular las disposiciones en red cuadrada y hexagonal de postes dieléctricos o huecos aéreos, dichos cristales son mostrados en la Fig. 3 con su celda de Wigner-Seitz (caso cuadrado) y su celda unitaria (caso hexagonal) encerradas por líneas rojas.

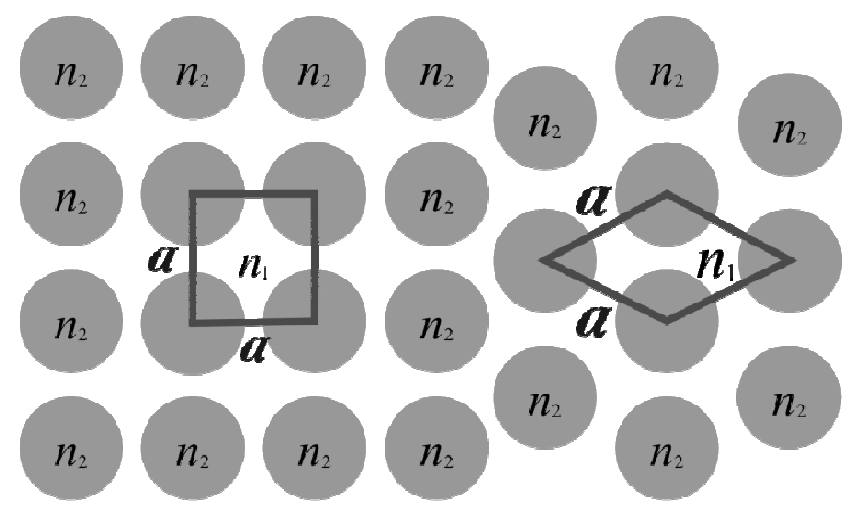

Fig. 3. CRistal Fotónico 2D EN RED CUADRADA Y HEXAgonal CON CELDA UNITARIA ENTRE LÍNEAS ROJAS DE LONGITUD $a$ 
Las matrices $\boldsymbol{D}_{x}^{E_{z}}, \boldsymbol{D}_{x}^{H_{y}}, \boldsymbol{D}_{y}^{H_{x}}, \boldsymbol{D}_{y}^{E_{z}}, \boldsymbol{E}_{z}$ y $\boldsymbol{\epsilon}$ asociadas al cristal 2D de red cuadrada con un grid de 2 x 2 puntos son:

$$
\begin{gathered}
\boldsymbol{D}_{x}^{E_{z}}=\frac{1}{\Delta l}\left(\begin{array}{cccc}
-1 & 1 & 0 & 0 \\
e^{i k_{x} a} & -1 & 0 & 0 \\
0 & 0 & -1 & 1 \\
0 & 0 & e^{i k_{x} a} & -1
\end{array}\right) \boldsymbol{E}_{z}=\left(\begin{array}{c}
E_{z}^{11} \\
E_{z}^{12} \\
E_{z}^{21} \\
E_{z}^{22}
\end{array}\right) \\
\boldsymbol{D}_{x}^{H_{y}}=-\left(\boldsymbol{D}_{x}^{E_{z}}\right)^{\dagger} \\
\boldsymbol{D}_{y}^{E_{z}}=\frac{1}{\Delta l}\left(\begin{array}{cccc}
-1 & 0 & 1 & 0 \\
0 & -1 & 0 & 1 \\
e^{i k_{y} a} & 0 & -1 & 0 \\
0 & e^{i k_{y} a} & 0 & -1
\end{array}\right) \\
\boldsymbol{D}_{y}^{H_{y}}=-\left(\boldsymbol{D}_{y}^{E_{z}}\right)^{\dagger} \\
\boldsymbol{\epsilon}=\left(\begin{array}{cccc}
\epsilon^{11} & 0 & 0 & 0 \\
0 & \epsilon^{12} & 0 & 0 \\
0 & 0 & \epsilon^{21} & 0 \\
0 & 0 & 0 & \epsilon^{22}
\end{array}\right)
\end{gathered}
$$

Donde $\epsilon^{i j}=\epsilon(i \Delta l, j \Delta l), H_{y}^{i j}=H_{y}(i \Delta l, j \Delta l), \Delta l=\Delta x=\Delta y$ y $e^{i k_{j} a}$ representa la condición de frontera de Bloch (19). Para el caso en el que la red es hexagonal las matrices en un grid de $2 \mathrm{x} 1$ son:

$$
\begin{gathered}
\boldsymbol{D}_{x}^{E_{z}}=\frac{1}{\Delta x}\left(\begin{array}{cc}
-1 & 1 \\
e^{i a k_{x}} & -1
\end{array}\right) \boldsymbol{E}_{z}=\left(\begin{array}{c}
E_{z}^{11} \\
E_{z}^{21}
\end{array}\right) \\
\boldsymbol{D}_{x}^{H_{y}}=-\left(\boldsymbol{D}_{x}^{E_{z}}\right)^{\dagger} \\
\boldsymbol{D}_{y}^{E_{z}}=\frac{1}{\Delta y}\left(\begin{array}{cc}
-1 & -1 \\
e^{i\left(0.5 a k_{x}+0.5 \sqrt{3} a k_{y}\right)} & e^{-i a k_{x}+i\left(0.5 a k_{x}+0.5 \sqrt{3} a k_{y}\right)}
\end{array}\right) \\
\boldsymbol{D}_{y}^{H_{y}}=-\left(\boldsymbol{D}_{y}^{E_{z}}\right)^{\dagger} \\
\boldsymbol{\epsilon}=\left(\begin{array}{cc}
\epsilon^{11} & 0 \\
0 & \epsilon^{21}
\end{array}\right)
\end{gathered}
$$

Donde $\epsilon^{i j}=\epsilon(i \Delta x, j \Delta y), H_{y}^{i j}=H_{y}(i \Delta x, j \Delta y) \mathrm{y} e^{-i a k_{x}+i\left(0.5 a k_{x}+\right.}$ $\left.0.5 \sqrt{3} a k_{y}\right), \mathrm{y} e^{i\left(0.5 a k_{x}+0.5 \sqrt{3} a k_{y}\right)}$ representan las condiciones de frontera de Bloch (19). Una vez conocidas estas matrices se cons- 
truyen los operadores matriciales $\boldsymbol{\epsilon}^{-\mathbf{1}} \boldsymbol{D}_{x}^{H_{y}} \boldsymbol{D}_{x}^{E_{z}}$ y $\boldsymbol{\epsilon}^{-\mathbf{1}}\left(\boldsymbol{D}_{x}^{H_{y}} \boldsymbol{D}_{x}^{E_{z}}+\boldsymbol{D}_{y}^{H_{x}} \boldsymbol{D}_{y}^{E_{z}}\right)$, La diagonalización numérica de dichos operadores para cada $k_{x} \mathrm{y}$ $k_{y}$ permitirá hallar los autovalores del sistema (energías) y sus autovectores (campos) (Rumpf, 2006).

\section{Simulación y RESUltados}

La ecuación (16) fue diagonalizada sucesivamente para los valores de comprendidos en el intervalo $[-\pi, \pi]$ (primera zona de Brillouin), en la Fig. 4 se muestra el resultado de esta simulación, que corresponde al diagrama de bandas del cristal fotónico superpuesto con el diagrama de bandas calculado para el mismo sistema con el software MPB (Johnson, 2004). Este cristal está compuesto por pares de difractores de Bragg con constantes dieléctricas $\boldsymbol{\epsilon}_{1}=12.25$ y $\boldsymbol{\epsilon}_{2}=4$ respectivamente y longitudes de $0.5 a$.

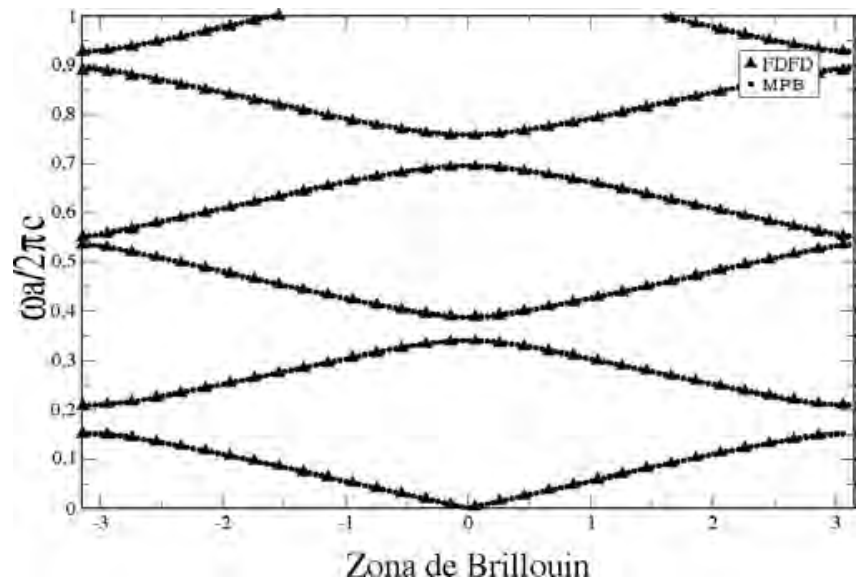

Fig. 4. Diagrama de bandas para un Cristal fotónico $1 \mathrm{D}$ de $\boldsymbol{\epsilon}_{1}=12.25$ y $\boldsymbol{\epsilon}_{2}=4$, CELDA UNITARIA DE LONGITUD $a$ Y DIFRACTORES DE LONGITUD

Un defecto fue introducido en el cristal fotónico aumentando la longitud de una capa de $\boldsymbol{\epsilon}_{1}=12.25$ hasta un tamaño de 1.5a. Para realizar cálculos con sistemas defectivos utilizando la condición de 
frontera de Bloch es necesario devolverle la periodicidad al cristal fotónico, esta tarea es llevada a cabo utilizando la técnica de la supercelda (Wu et al., 2003), que considera una celda unitaria mucho más grande que la del cristal regular pero con el defecto ubicado en el centro y pares de difractores de Bragg alrededor de éste. La replicación periódica de la supercelda reproducirá un cristal con un defecto periódico que será más cercano al sistema con un sólo defecto cuanto más pares de difractores hayan entre defectos sucesivos.

La Fig. 5 muestra un cálculo de diagrama de bandas para el cristal con defecto y el perfil de intensidad del modo que se localiza en dicho defecto superpuesto con la función dieléctrica de la supercelda. En el diagrama de bandas de esta figura se nota la clara penetración de un modo a la zona de gap, además, es de notar que aparecen nuevas bandas en este diagrama, esto es producido debido al uso de una celda que no es unitaria, por lo que la zona de Brillouin se reduce y comprime las bandas a través de un doblamiento de éstas (Wu et al., 2003).
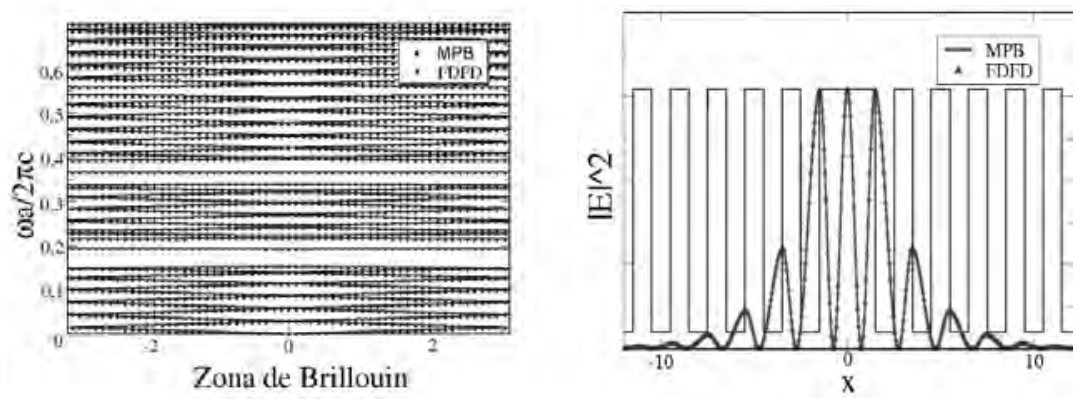

Fig. 5. Diagrama de BANDAS PARA EL CRISTAL 1D CON DEFECTO SUPERPUESTO CON EL CALCULADO EN MPB, Y PERFIL DE INTENSIDAD DEL MODO LOCALIZADO EN DICHO DEFECTO

Los cálculos $2 \mathrm{D}$ se hicieron a través de la diagonalización de (17), pues es en el caso TM donde aparece band-gap para ambos tipos de red, cuadrada y triangular. Sin embargo, para ciertos valores de constante dieléctrica y radio de los cilindros dieléctricos es posible obtener band-gap completo en la red triangular (TM y 
TE) (Joannopoulos et al., 2008). Para la red cuadrada la diagonalización se realizó sucesivamente en el borde de la primera zona de Brillouin reducida delimitada por lo puntos de simetría $\Gamma, \mathrm{X}$ y M (Joannopoulos et al., 2008), el resultado de esta simulación es mostrado en la Fig. 6 para cilindros de radio $0.2 a$ y constante dieléctrica $\boldsymbol{\epsilon}=8.9$, también es superpuesto el diagrama de bandas calculado con MPB.

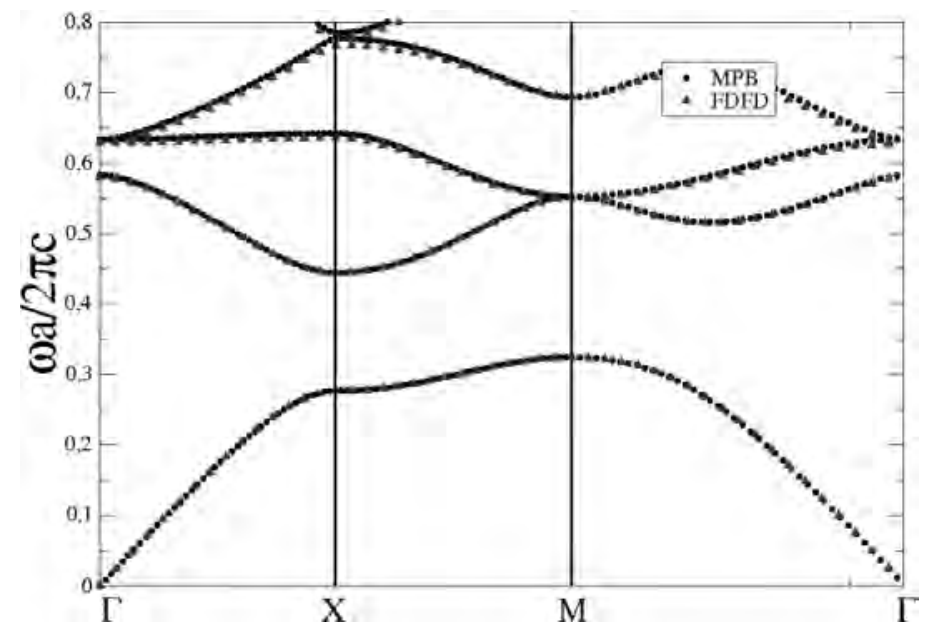

Fig. 6. DiAgRAMA DE BANDAS PARA EL CASO TM DE UNA RED CUADRADA CON CILINDROS DE RADIO $0.2 a$ Y $\boldsymbol{\epsilon}=8.9$

Los perfiles de intensidad del campo eléctrico también fueron calculados para esta estructura en los puntos de simetría X y M en la primera y segunda banda (base y excitado), estos son mostrados en la Fig. 7 junto con el campo eléctrico calculado en MPB. 


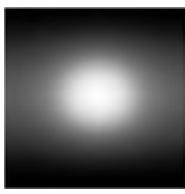

$\mathrm{X}-1$ (FDFD)

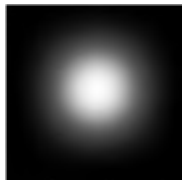

M-1 (FDFD)

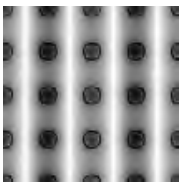

$\mathrm{X}-1$ (MPB)

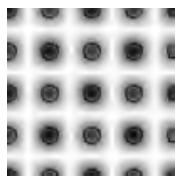

M-1 (MPB)

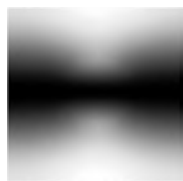

$\mathrm{X}-2$ (FDFD)

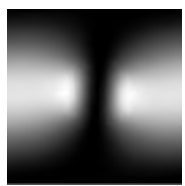

M-2 (FDFD)

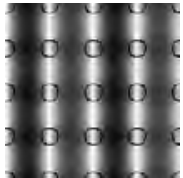

$\mathrm{X}-2$ (MPB)

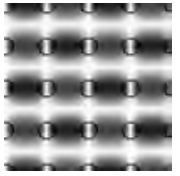

M-2 (MPB)

Fig. 7. Perfiles de intensidad calculados con FDFd (1 PeRiodo), y CAMpo eléctrico CALCULADO CON MPB (4 PERIODOS)

Para la red triangular, al igual que en la red cuadrada, la diagonalización se realizó sucesivamente en el borde de la zona de Brillouin reducida delimitada por los puntos de simetría $\Gamma, \mathrm{M}$ y K. El resultado de dicha simulación se muestra en la Fig. 8 para una estructura con cilindros de radio $0.2 a$ y constante dieléctrica $\epsilon=12$, allí también se superpone el diagrama de bandas calculado en MPB.

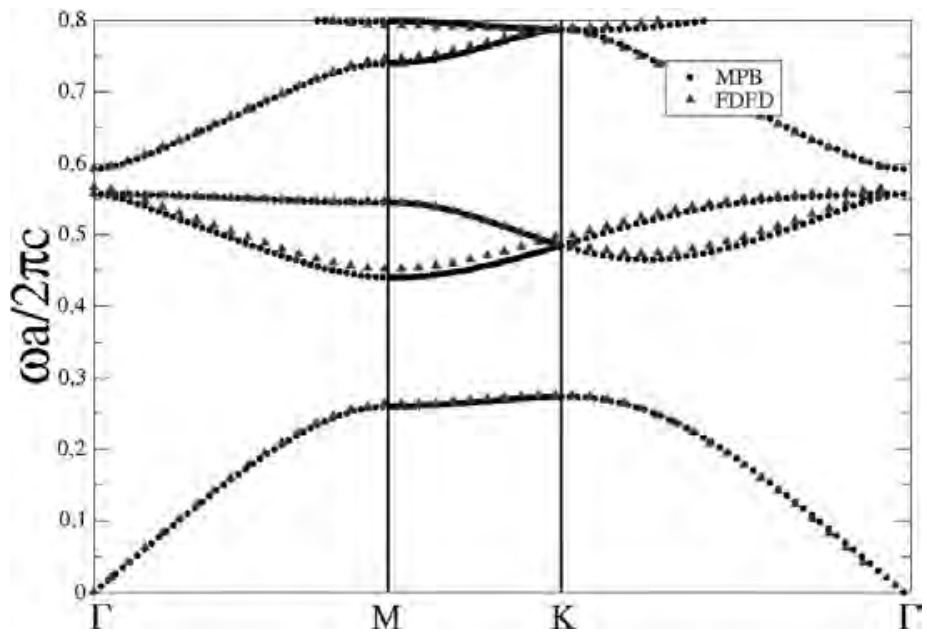

Fig. 8. DIAGRAMA DE BANDAS PARA EL CASO TM DE UNA RED TRIANGULAR CON CILINDROS DE RADIO $0.2 a$ Y $\boldsymbol{\epsilon}=12$ 
Los perfiles de intensidad de campo eléctrico (base y excitado) fueron calculados en los puntos de simetría M y K, y son mostrados en la Fig. 9 junto con los respectivos calculados en MPB.

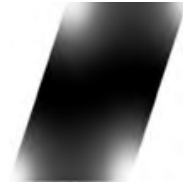

M-1 (FDFD)

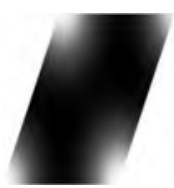

K-1 (FDFD)

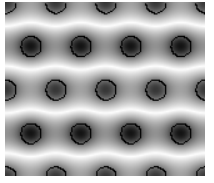

M-1 (MPB)

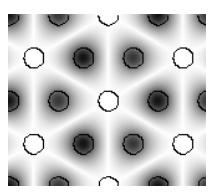

$\mathrm{K}-1$ (MPB)

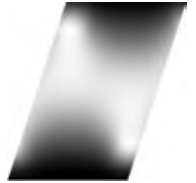

M-2 (FDFD)

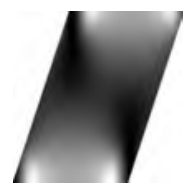

K-2 (FDFD)

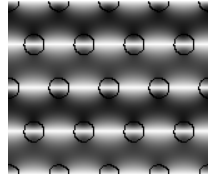

M-2 (MPB)

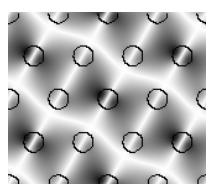

K-2 (MPB)

Fig. 9. Perfiles de intensidad Calculados Con FDFd (1 Periodo), Y CAMPo eléctrico CALCULADO CON MPB (4 PERIODOS)

\section{Discusıón}

Los resultados anteriores fueron obtenidos a través de una diagonalización en un grid de 200 puntos para el caso 1D, 400 puntos para el caso $2 \mathrm{D}$ cuadrado y 800 puntos para el caso $2 \mathrm{D}$ hexagonal. Estos valores se discriminaron realizando sucesivas simulaciones hasta obtener una comparación aceptable con los resultados obtenidos en MPB. Las Fig. 4, 6 y 8 evidencian la aparición de band-gaps o regiones energéticamente prohibidas en la estructura, por lo que una onda electromagnética que trate de propagarse con energía en dicho rango decaerá exponencialmente en el cristal.

Estas regiones prohibidas aparecen debido a fenómenos de interferencia destructiva gobernados por la ley de Bragg (Yariv $\&$ Yeh, 2007). Una vez un defecto es introducido en la estructura (tanto en cristales 1D como 2D y 3D) es posible producir la penetración de uno o varios modos en el gap del cristal fotónico, esto se evidencia en la Fig. 5 donde un modo penetra a esta zona, 
[140] Método de diferencias finitas en el dominio de las frecuencias para cristales fotónicos...

localizándose en la zona defectuosa tal y como es mostrado en el perfil de intensidad junto al diagrama de bandas. Las Fig. 7 y 9 evidencian que las concentraciones de campo está en las regiones de mayor dieléctrico en el modo base, y las concentraciones migran a las regiones de menos dieléctrico para el modo excitado, este hecho es esperado en un cristal fotónico y es una consecuencia del principio variacional aplicado a estos sistemas (Joannopoulos et $a l .$, 2008).

\section{Conclusiones}

El método de diferencias finitas en el dominio de las frecuencias es un método de fácil implementación, que permite realizar estudios numéricos en un cristal fotónico de una forma rápida y eficiente. Las dos grandes ventajas que otorga FDFD es la flexibilidad que tiene al estudiar cristales con funciones dieléctricas cuya celda unitaria tenga objetos exóticos en su interior (pues dichos objetos son introducidos a través de una función definida a tramos) y la baja demanda de recursos computacionales, diferente de métodos como FDTD, donde el tiempo también es discretizado y el consumo de recursos computacionales es elevado.

Para un cristal fotónico 1D, un grid de 70 puntos por unidad de longitud fue suficiente para alcanzar una convergencia adecuada de las energías del sistema, y para cristales $2 \mathrm{D}$ un grid de 400 puntos (en el caso cuadrado) y uno de 800 puntos (en el caso hexagonal) por unidad de área, fue suficiente para obtener resultados comparables con los obtenidos en el software MPB. Es importante mencionar que el método FDFD se vuelve inestable cuando el número de puntos es muy elevado, por lo que aumentar la fineza de la malla no necesariamente produce mejores resultados, sin embargo, existen formas de optimizar el método para evitar elevar mucho el número de puntos en estructuras complejas (Taflove \& Hagness, 2000). 


\section{REFERENCIAS}

Chen, H., Chan, C.T., Sheng, P., (2010); Transformation optics and metamaterials, Nature Materials, 9, 387-396.

Joannopoulos, J.D., Villeneuve, P.R., Fan, S., (1997); Photonic crystals: putting a new twist on light, Nature, 386, 143-149.

Joannopoulos, J.D., Johnson, S.G., Winn, J.N., Meade, R.D., (2008); Photonic Crystals: Molding the Flow of Light, Princeton University Press, USA.

Johnson, S.G., (2004); MPB: MIT Photonic Bands. Consultado el 14 de Marzo de 2010, de http://ab-initio.mit.edu/wiki/index.php/MIT_PhotonicBands.

Kittel, C., (1996); Introduction to Solid State Physics, New York, John Wiley and Sons.

Lourtioz, J.M., (2005); Photonic Crystals: Towards Nanoscale Photonic Devices, Berlin Heidelberg, Springer.

Rumpf, R.C., (2006); Design and optimization of nano-optical elements by coupling fabrication to optical behavior (PhD. Thesis), Florida, University of Central Florida.

Sakoda, K., (2005); Optical Properties of Photonic Crystals, New York, Springer.

Taflove, A., Hagness, S.C., (2000); Computational Electrodynamics: The Finite-Difference Time-Domain Method, London Norwood MA, Artech House.

Vahala, K.J., (2003); Optical microcavities, Nature, 424, 839-846.

Wu, L., Zhuang, F., He, S., (2003); Degeneracy analysis for a supercell of a photonic crystal and its applications to the creations of band gaps, Physical Review, 67, 026612.

Yariv, A., Yeh, P., (2007); Photonics: Optical Electronics in Modern Communications, USA, Oxford University Press.

Yee, K.S., (1966); Numerical solution of the initial boundary value problems involving Maxwell's equations in isotropic media, IEEE Trans. Antennas and Propagations, 14, 302-307. 